\title{
Interfacial Shear Stresses Calculation of FRP Strengthened RC Beams
}

\author{
Jia Yang ${ }^{12, \text { a }}$ \\ ${ }^{1}$ College of Architectural and Civil Engineering, Shenyang University, Shenyang, China \\ ${ }^{2}$ The Key Laboratory of GeoenvironMental Engineering, Liaoning, China \\ ayangjia0918@163.com
}

Keywords: Interfacial shear stresses; FRP plate; Reinforced concrete

Abstract. One major problem when using fiber reinforced polymer (FRP) to strengthen structures is the presence of high interfacial shear stresses near the end of the RC beams which might impact on the debonding failure modes and working mechanism. Interfacial debonding failure may occur at the plate ends due to interfacial stresses. This study analyzes the effect of the interfacial behavior on the interfacial shear stresses of FRP strengthened RC beams under uniformly distributed loads. The analytical model is established, and the differential equation of interfacial shear stresses of FRP strengthened RC beams is established. The calculated formula of interfacial shear stresses is deduced under uniformly distributed loads.

\section{Introduction}

With the development of structural adhesives and FRP in recent years, FRP-bonding techniques are now recognized as an effective, convenient, and economical method to reinforce concrete structures. It is well known that the FRP offers high reliability and durability and fatigue resistance as well as its higher strength to weight ratio. FRP plates can be bonded to the surface of RC beam through the structural adhesive. Then the FRP plate, concrete beam and adhesive layer work together. In this strengthening method, the performance of the FRP-concrete interface in providing an effective stress transfer is of crucial importance. However, the problem that arises when using this technique is the development of high interfacial shear stresses at the plate ends. Previous studies have shown that interfacial debonding failure will occur at the plate ends due to interfacial stresses. The mechanical properties and failure mechanism of strengthened member are closely related to bond performance of FRP-concrete interface ${ }^{[1-9]}$. So the research about the interfacial shear stresses of the FRP-concrete interface is necessary. Under external loading, the relative slip occurred on the interface between FRP plate and concrete causes the redistribution of stress and then influences mechanical behavior of component. Therefore, this paper studies on the shear stresses of FRP-concrete interface. Based on the analytical model of the interface between FRP and concrete, the differential equation of interfacial shear stresses of FRP strengthened RC beams is established. And the calculated formula of interfacial shear stresses is deduced under uniformly distributed loads.

\section{Analytical Model}

\section{Basic Assumptions}

A differential section $d x$, can be cut out from the FRP strengthened RC beams as shown in Fig.1. The composite beam is made from three materials: RC concrete, adhesive layer and FRP plate. According to the characteristics and mechanical behavior of FRP strengthened RC beams, we make 
the following assumptions for simplicity of the problems before starting the derivations:

1. Adherends are homogeneous and linear elastic;

2. Deformations of FRP plate and concrete are due to bending moments, axial and shear forces;

3. The adhesive layer is assumed to be subject to stresses invariant across its thickness;

4. The curvatures of FRP plate and concrete beam are assumed to be the same.

\section{Governing Differential Equation}

Considering a typical infinitesimal unit body of the FRP strengthened RC beams as shown in Fig.1.

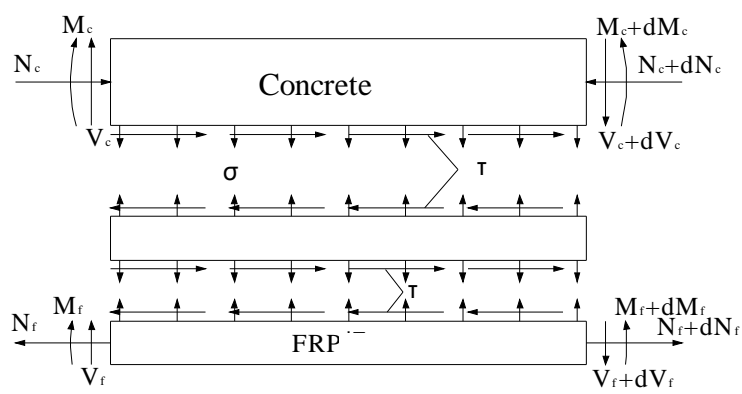

Fig.1 The force graph of unit body

Ignore the axial force, bending moment and shear force of adhesive layer, so the adhesive layer is exposed only to the interfacial shear and normal stresses. Adhesive layer is mainly transmitted shear force and shear deformation. $u(x, y)$ and $v(x, y)$ are the horizontal and vertical displacements respectively at any point in the adhesive layer as defined in Fig.1. The interfacial shear stresses are denoted by $\tau(x)$. The corresponding shear stress is given as follows.

$$
\tau(x)=G_{a}\left(\frac{d u(x, y)}{d y}+\frac{d v(x, y)}{d x}\right)
$$

Where $G_{a}$ is the shear modulus of the adhesive layer.

According to the basic assumptions, Eq. 2 can be rewritten as follow.

$$
\frac{d u(x, y)}{d y}=\frac{1}{t_{a}}\left(u_{f}(x)-u_{c}(x)\right)
$$

Where $u_{c}(x)$ and $u_{f}(x)$ are the longitudinal displacements at the base of concrete beam and the top of FRP plate respectively, and $t_{a}$ is the thickness of the adhesive layer.

Substituting Eq. 2 into Eq.1. Eq. 3 can be rewritten as follow.

$$
\frac{d \tau(x)}{d x}=G_{a}\left[\frac{1}{t_{a}}\left(\frac{d u_{f}(x)}{d x}-\frac{d u_{c}(x)}{d x}\right)+\frac{d^{2} v(x, y)}{d x^{2}}\right]
$$

The formula of $\frac{d^{2} v(x, y)}{d x^{2}}$ in Eq.3 is very small and thus is ignored in the following derivation. Eq. 4 can be rewritten as follow.

$$
\frac{d \tau(x)}{d x}=\frac{G_{a}}{t_{a}}\left(\varepsilon_{f}(x)-\varepsilon_{c}(x)\right)
$$

Where $\varepsilon_{f}(x), \varepsilon_{c}(x)$ are the strain at the bottom of concrete beam and the top of FRP plate respectively.

Considering axial and bending deformations, the formulas are given as below. 


$$
\begin{aligned}
& \varepsilon_{c}(x)=\frac{M_{c}(x) y_{1}}{E_{c} I_{c}}-\frac{N_{c}(x)}{E_{c} A_{c}} \\
& \varepsilon_{f}(x)=-\frac{M_{f}(x) y_{2}}{E_{f} I_{f}}+\frac{N_{f}(x)}{E_{f} A_{f}}
\end{aligned}
$$

Where $t_{f}, b_{f}$ are the thickness and width of the FRP plate respectively; $E_{c}, E_{f}, I_{c}, I_{f}$ are elastic modulus, inertia moments of RC beam and FRP plate respectively; $A_{c}, A_{f}$ are cross-sectional areas of RC beam and FRP plate respectively; $M_{c}(x), M_{f}(x), N_{c}(x), N_{f}(x), V_{c}(x), V_{f}(x)$ are the bending moment, axial and shear forces in each adherend while $y_{1}$ and $y_{2}$ are the distances from the bottom of RC beam and the top of FRP plate to their respective centroid. The following equilibrium equations are established as below.

$$
\begin{aligned}
& \frac{d M_{f}(x)}{d x}=V_{f}-\tau b_{f} y_{2} \\
& \frac{d M_{c}(x)}{d x}=V_{c}-\tau b_{f} y_{1} \\
& \frac{d N_{c}(x)}{d x}=\frac{d N_{f}(x)}{d x}=\tau b_{f}
\end{aligned}
$$

Assuming equal curvature in the concrete beam and FRP plate, the relationship between the moments in the two adherends can be expressed as below.

$$
\frac{M_{f}(x)}{E_{f} I_{f}}=\frac{M_{c}(x)}{E_{c} I_{c}}
$$

Substituting Eq. 5-10 into Eq.4, and get derivative, then the differential equation refers with Eq.11.

$$
\frac{d^{2} \tau(x)}{d x^{2}}=\alpha^{2} \tau-\beta V(x)
$$

Where $\quad \alpha^{2}=\frac{G_{a}}{t_{a}} b_{f}\left[\frac{1}{E_{f} A_{f}}+\frac{1}{E_{c} A_{c}}+\frac{\left(y_{1}+y_{2}\right)^{2}}{E I}\right], \quad \beta=\frac{G_{a}\left(y_{1}+y_{2}\right)}{E I t_{a}} \quad, \quad E I=E_{f} I_{f}+E_{c} I_{c} \quad$ and $V(x)=V_{f}+V_{c}$.

\section{Calculation formulas}

Uniformly distributed loads are $q$. RC beam length is $L$. The length of FRP plate is $l_{f}$. The calculating diagram under uniformly distributed loads is shown in Fig.2.

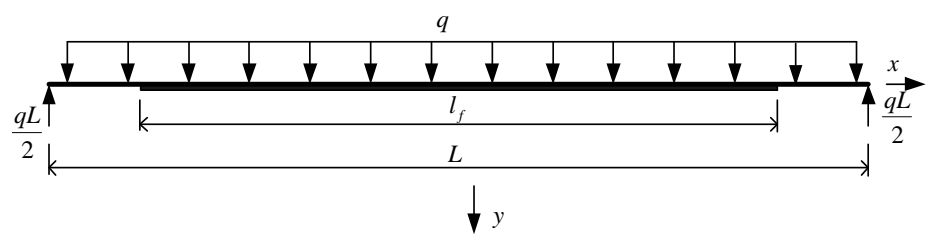

Fig.2. Uniformly distributed loads

Under external loading, the moment of beam section is established as below. 


$$
M(x)=\frac{q}{8}\left(L^{2}-4 x^{2}\right) \quad\left(-\frac{L}{2} \leq x \leq \frac{L}{2}\right)
$$

The solution to Eq.11 can be obtained by using the following boundary conditions.

When $x=0$, there is $\tau=0$ and when $x=0.5 l_{f}$, there is $\frac{d \tau}{d x}=-\frac{G_{a} q\left(L^{2}-l_{f}^{2}\right)\left(y_{1}+y_{2}\right)}{8 E I t_{a}}$. The calculated formula of interfacial shear stresses under uniformly distributed loads refers with Eq.13.

$$
\tau(x)=\frac{8 E I_{a} \beta q-G_{a} q \alpha^{2}\left(L^{2}-l_{f}^{2}\right)\left(y_{1}+y_{2}\right)}{8 E \operatorname{EIt}_{a} \alpha^{3}\left(e^{\frac{1}{2} \alpha l_{f}}+e^{-\frac{1}{2} \alpha l_{f}}\right)}\left(e^{\alpha x}-e^{-\alpha x}\right)-\frac{\beta q}{\alpha^{2}} x
$$

\section{Conclusions}

Based on the analytical model, the differential equation of interfacial shear stresses of FRP strengthened RC beams is established. And the calculated formula of interfacial shear stresses is deduced under uniformly distributed loads. The calculation formula of interfacial shear stresses is rational in theory, but the applicability of the calculation formula needs more experiments to verify.

\section{References}

[1] Lianguang Wang. Prestressed Composite Structures of Steel and Concrete. Beijing Science Press (2009) in press. (In Chinese)

[2] A.S. Bouchikhi, A. Lousdad, A. Megueni. On the reduce of interfacial shear stresses in fiber reinforced polymer plate retrofitted concrete beams. Materials and Design, 31 (2010) 1508-1515

[3] Teng J. G, Yuan H. FRP-to concrete interface between two adjacent cracks: Theoretical model for debonding failure. International Journal of Solids and Structures, Vol. 43(2006), p. 5750.

[4] Benachour A., Benyoucef S. Interfacial stress analysis of steel beams reinforced with bonded prestressed FRP plate. Engineering Structures, Vol. 30(2008), p. 3305.

[5] A. Lousdad, A. Megueni, A.S. Bouchikhi. Geometric edge shape based optimization for interfacial shear stress reduction in fiber reinforced polymer plate retrofitted concrete beams. Computational Materials Science, 47 (2010): 911-918

[6] Tim S., John C. Elastic analysis of adhesion stress for the design of a strengthening plate bonded to a beam. Construction and Building Materials, Vol. 20(2006), p. 34.

[7] Fangliang Chen, Pizhong Qiao. Debonding analysis of FRP-concrete interface between two balanced adjacent flexural cracks in plated beams. International Journal of Solids and Structures, Vol. 46 (2009),p.2618-2628.

[8] S.T. Smith, J.G. Teng. Interfacial stresses in plated beams. Engineering Structures 23 (2001) 857-871.

[9] Zhang Jiwen, Lu Zhitao, Teng Jinguang, Smith S. T. Analysis of the interfacial stresses of the strengthened RC beams externally bonded with CFRP sheets or steel plates. Industrial Construction, 2001, 31(6): 1-4. (In Chinese) 